\title{
Xantomas tuberosos en un paciente pediátrico: la clave en el diagnóstico precoz de una patología grave y poco frecuente
} Tuberous xanthomas in a pediatric patient: a clue to the early diagnosis of a severe and infrequent disease

\author{
Loreto Heredia $^{a}$ (D), Javier Arellano ${ }^{a, b}$ (D), Yamile Corredoira ${ }^{c, d}$ (D)
}

\section{RESUMEN}

Los xantomas cutáneos reflejan el depósito de lípidos en la piel y pueden ser la única manifestación temprana de dislipidemias de inicio en la infancia. Las características y distribución de los xantomas orientan a la patología de base; los xantomas tuberosos tienen una fuerte asociación con la hipercolesterolemia homocigota familiar, una patología muy infrecuente. Su detección temprana otorga una ventana terapéutica para prevenir la ateroesclerosis acelerada y la mortalidad. Se presenta el caso de una paciente que comenzó a los dos años con xantomas tuberosos, que fueron la clave diagnóstica para identificar la hipercolesterolemia homocigota familiar subyacente.

Palabras clave: xantomas; hipercolesterolemia familiar.

\begin{abstract}
Cutaneous xanthomas reflect lipid deposition on the skin and may be the only early manifestation of a childhoodonset dyslipidemia. Characteristics and distribution of the xanthomas signalize the underlying pathology, tuberous xanthomas being strongly associated with homozygous familial hypercholesterolaemia, an extremely rare condition. Its early detection provides a therapeutic window to prevent accelerated atherosclerosis and mortality. We present the case of a patient who started at two years with tuberous xanthomas, which were the diagnostic clue to identify the underlying homozygous familial hypercholesterolaemia.

Key words: xanthomas; familial hypercholesterolemia.
\end{abstract}

http: / / dx.doi.org/10.5546/ aap.2022.e34

Cómo citar: Heredia L, Arellano J, Corredoira Y. Xantomas tuberosos en un paciente pediátrico: la clave en el diagnóstico precoz de una patología grave y poco frecuente. Arch Argent Pediatr 2022;120(1):e34-e38.

a. Departamento de Dermatología, Facultad de Medicina, Universidad de Chile, Santiago, Chile.

b. Servicio de Dermatología, Hospital Clínico San Borja Arriarán, Santiago, Chile.

c. Departamento de Anatomía Patológica, Facultad de Medicina, Universidad de Chile, Santiago.

d. Servicio de Anatomía Patológica, Hospital Clínico San Borja Arriarán, Santiago, Chile.

Correspondencia:

Javier Arellano: javierarlo@uchile.cl

Financiamiento: Ninguno.

Conflicto de intereses: Ninguno que declarar.

Recibido: 13-1-2021

Aceptado: 16-7-2021

\section{INTRODUCCIÓN}

Los xantomas son depósitos localizados de lípidos que se pueden manifestar a nivel cutáneo como pápulas, placas o nódulos de color amarillo-anaranjados. Pueden ser idiopáticos o ser manifestación de dislipidemias primarias o secundarias. El tipo de xantomas también otorga una clave para el diagnóstico de la anormalidad subyacente. ${ }^{1}$ La presencia de xantomas tuberosos, especialmente en los niños, tiene una fuerte asociación con la hipercolesterolemia homocigota familiar (HHF); ; ${ }^{1,2}$ esto permite orientar hacia el diagnóstico precoz e iniciar un tratamiento que evite la ateroesclerosis acelerada y los eventos cardiovasculares tempranos, que, sin tratamiento, podrían ocurrir en la infancia. ${ }^{2}$

Se presenta el caso de una paciente que inició a los dos años con xantomas tuberosos que llevaron al diagnóstico de una HHF.

\section{CASO CLÍNICO}

Paciente de sexo femenino de cuatro años de edad, sin antecedentes mórbidos, hija de padres no consanguíneos. Fue evaluada en atención primaria por lesiones de dos años de evolución, de aparición progresiva en rodillas, muñecas y región interglútea, asintomáticas y diagnosticadas inicialmente como verrugas vulgares. Se le realizó extirpación por electrocirugía. Luego del procedimiento, evolucionó con placas induradas persistentes cubiertas por costras (Figura 1), que curaron con recidiva de las lesiones, por lo que fue derivada a dermatología.

Al momento de la evaluación en el servicio se observaban múltiples nódulos amarillos, de consistencia firme, superficie lisa y confluentes en ambas rodillas, muñecas y región interglútea. Además, se observó un nódulo único de similares características en el quinto espacio interdigital de la mano derecha (Figura 1). El examen histológico reveló un infiltrado inflamatorio leve, células espumosas, células multinucleadas de Touton, fibroblastos aumentados y depósito de colágeno. Compromiso hipodérmico por células espumosas 
en la dermis (Figura 2), lo que confirmó el diagnóstico de xantomas tuberosos.

Se solicitó un perfil lipídico, en el que se destacaron valores de colesterol total de $849 \mathrm{mg} /$ dl, LDL de $770 \mathrm{mg} / \mathrm{dl}$, HDL de $39 \mathrm{mg} / \mathrm{dl} \mathrm{y}$ triglicéridos de $198 \mathrm{mg} / \mathrm{dl}$; el perfil hepático, el perfil renal y la glucemia resultaron normales. Ambos padres tenían historia de hipercolesterolemia en tratamiento. Sus dos hermanas mayores, de 9 y 13 años, no presentaban lesiones cutáneas y se estudiaron con perfil lipídico, que estaba dentro de valores normales. Se realizó estudio genético a la paciente, en el que se confirmó una mutación homocigota del gen del receptor de LDL. El estudio cardiovascular resultó normal.

Se indicaron pautas para el estilo de vida, con modificaciones en la dieta y actividad física. Se

FIgURA 1. Lesiones cutáneas en la paciente presentada. A: lesión luego de la electrocirugía; se observan placas induradas, cubiertas por costras, en ambas rodillas. B: xantoma interglúteo. C: xantoma interdigital. D: xantomas tuberosos en ambas rodillas

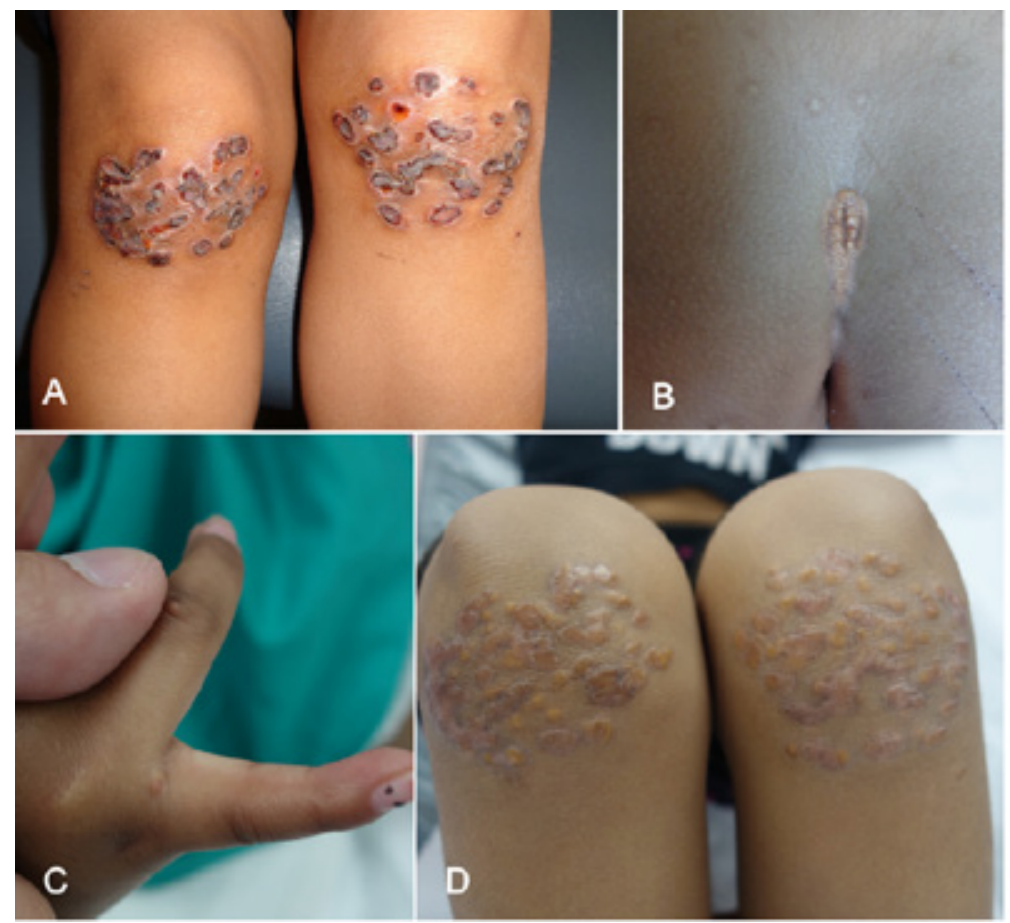

FiguRA 2. Imágenes histológicas de un xantoma de la rodilla. Se observa un infiltrado inflamatorio leve, células espumosas, células multinucleadas de Touton, fibroblastos aumentados y depósito de colágeno en la dermis. En la hipodermis también se observa la presencia de células espumosas

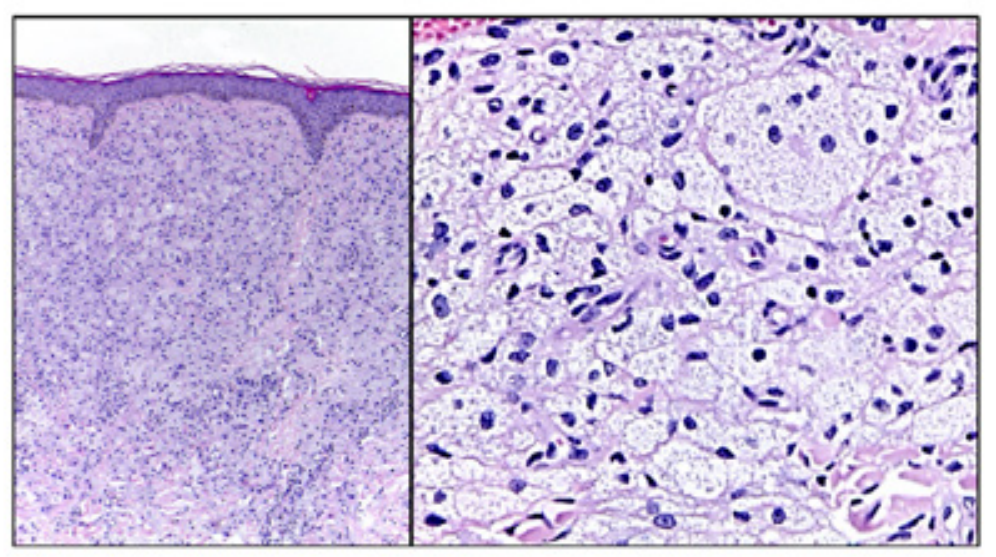


indicó tratamiento farmacológico por vía oral con ezetimiba ( $5 \mathrm{mg}$ ) asociado a atorvastatina (5 mg). Debido a la falta de respuesta, se aumentó la dosis hasta ezetimiba $10 \mathrm{mg}$ y atorvastatina $20 \mathrm{mg}$. La paciente no ha regresado a controles a dermatología, pero en el control con endocrinología se constató una mejora parcial del perfil lipídico con manejo intensivo de la dieta y administración de los fármacos a dosis máximas.

\section{DISCUSIÓN}

Los xantomas cutáneos son una manifestación de depósito de lípidos en la dermis y se presentan como pápulas, placas o nódulos firmes de color amarillo anaranjado. La histología revela la presencia de células espumosas, que corresponden a macrófagos que han fagocitado los lípidos depositados en los tejidos. La mayoría de los xantomas cutáneos aparecen en la edad adulta, excepto en un número acotado de patologías de inicio en la infancia, de las cuales la más frecuente es la HHF, que corresponde al caso clínico presentado. ${ }^{13,4}$ La morfología y la localización anatómica de los xantomas orienta el trastorno subyacente (Tabla 1).

En la paciente aquí presentada, las manifestaciones cutáneas fueron xantomas tuberosos y xantomas intertriginosos de aparición en la infancia temprana, cuadro clínico que por sí solo sugería el diagnóstico de HHF. Se destaca la ausencia de xantomas tendinosos que se describen con frecuencia en estos pacientes. ${ }^{5}$

La hipercolesterolemia familiar es una patología de herencia autosómica dominante, con mutaciones en el gen que codifica al receptor de LDL (LDLR). Para su forma heterocigota, se estima una prevalencia de 1 / 500 personas, se manifiesta en la edad adulta con alteraciones en el perfil lipídico o enfermedad coronaria prematura, y responde a la terapia con estatinas. ${ }^{6,7}$ La forma homocigota es muy infrecuente, con una prevalencia estimada de 1/1000 000 personas; presenta manifestaciones desde la infancia temprana ${ }^{7}$ con niveles de colesterol en sangre mayores de $500 \mathrm{mg} / \mathrm{dl}$ y ateroesclerosis prematura y progresiva. Estos cuadros requieren una intervención precoz para prevenir sus complicaciones. ${ }^{8}$ Sin tratamiento, se espera que los pacientes desarrollen ateroesclerosis antes de los 20 años, y la expectativa de vida es menor a 30 años. $^{8}$

Las manifestaciones cutáneas de la HHF son la clave clínica para llegar al diagnóstico precoz. Estas incluyen los xantomas cutáneos, xantomas tuberosos y los xantomas tendinosos, estos dos últimos con una fuerte asociación

TABLA 1. Tipo de xantomas y trastornos subyacentes ${ }^{1,3-6}$

\begin{tabular}{|c|c|c|}
\hline Tipo de lesión & Descripción clínica & Trastornos subyacentes \\
\hline Xantomas eruptivos & $\begin{array}{l}\text { Pápulas amarillentas, frecuentemente con halo } \\
\text { eritematoso, de } 1 \text { a } 5 \mathrm{~mm} \text {, que aparecen de forma súbita } \\
\text { y se agrupan en las superficies extensoras de las } \\
\text { extremidades y en glúteos Presentan fenómeno de } \\
\text { Koebner. }\end{array}$ & $\begin{array}{l}\text { Hipertrigliceridemia } \\
\text { primaria o secundaria. }\end{array}$ \\
\hline Xantomas tuberosos & $\begin{array}{l}\text { Nódulos firmes, de superficie lisa, que se localizan } \\
\text { en las superficies extensoras de los codos, rodillas, } \\
\text { nudillos y glúteos. }\end{array}$ & $\begin{array}{l}\text { Hipercolesterolemia, en } \\
\text { particular la forma } \\
\text { homocigota familiar. }\end{array}$ \\
\hline Xantomas tendíneos & $\begin{array}{l}\text { Nódulos subcutáneos firmes, con piel suprayacente } \\
\text { normal, que se encuentran en la fascia, ligamentos, } \\
\text { tendón de Aquiles y tendones extensores de manos, } \\
\text { codos y rodillas. }\end{array}$ & $\begin{array}{l}\text { Disbetalipoproteinemia } \\
\text { de tipo III. } \\
\text { Sitosterolemia. } \\
\text { Xantomatosis cerebrotendinosa. }\end{array}$ \\
\hline Xantomas planos & Pápulas o placas planas, de color amarillo anaranjadas. & \\
\hline Xantelasmas palpebrales & $\begin{array}{l}\text { Xantomas planos en los párpados y el ángulo interno } \\
\text { del ojo. }\end{array}$ & $\begin{array}{l}\text { Dislipidemias }(50 \%) \text { o } \\
\text { pacientes sanos }(50 \%)\end{array}$ \\
\hline Xantomas estriados palmares & Xantomas planos de los surcos palmares. & Disbetalipoproteinemia de tipo IIII. \\
\hline Xantomas intertriginosos & $\begin{array}{l}\text { Xantomas planos, con superficie en "empedrado", } \\
\text { en membranas interdigitales de las manos y } \\
\text { zonas intertriginosas. }\end{array}$ & $\begin{array}{l}\text { Hipercolesterolemia } \\
\text { homocigota familiar. }\end{array}$ \\
\hline Otras localizaciones & $\begin{array}{l}\text { Xantomas planos generalizados, en manos y pies, cuello, } \\
\text { tronco superior, pliegues de flexión y periorbitarios. }\end{array}$ & $\begin{array}{l}\text { Colestasia y gammapatía } \\
\text { monoclonal. }\end{array}$ \\
\hline
\end{tabular}


con la HHF cuando inician en la infancia. ${ }^{8}$ Los xantomas interdigitales en la infancia serían patognomónicos de esta condición. ${ }^{1,9}$ La presencia de arco corneal junto a estos hallazgos refuerza el diagnóstico clínico; no estaba presente en la paciente ni en sus padres. ${ }^{8}$ Estas manifestaciones cutáneas pueden revertir de manera lenta al corregir los niveles de colesterol en sangre, pero muchas veces requieren extirpación quirúrgica. ${ }^{3}$ El diagnóstico se confirma con el estudio genético; si este no estuviera disponible o si el caso plantea dudas, el diagnóstico puede apoyarse en el cuadro clínico, la historia familiar y el laboratorio (Tabla 2).

Tal como se describe, en el caso presentado las manifestaciones cutáneas llevaron al diagnóstico; sin embargo, llama la atención el transcurso de dos años desde el inicio de los síntomas y el manejo inicial como verrugas, que en parte puede deberse a la baja frecuencia con que se observan xantomas en la edad pediátrica. El diagnóstico se confirmó con el estudio genético, en un contexto en que la clínica, la historia familiar y el colesterol total y LDL muy elevados ya sugerían fuertemente el diagnóstico.

Con base en las últimas guías publicadas, el tratamiento de la HHF debe ser instaurado de manera precoz y su objetivo es reducir los niveles de colesterol LDL, idealmente a valores menores de $<135 \mathrm{mg} / \mathrm{dl}$, para evitar la ateromatosis y el daño cardiaco irreversible. ${ }^{10} \mathrm{~A}$ todos los pacientes se les debe indicar modificaciones en el estilo de vida, con dieta rica en frutas, vegetales y granos enteros, baja en grasas saturadas y colesterol, asociada a la actividad física. ${ }^{8}$

El manejo farmacológico clásico se basa en estatinas asociadas a ezetimibe. Esta combinación suele no ser suficiente, y es ineficaz si no hay actividad residual del LDLR. Se sugiere un ensayo terapéutico, con las dosis máximas toleradas de estos fármacos, para evaluar la respuesta.

La aféresis de lipoproteínas es un tratamiento adyuvante seguro, que logra la eliminación física de las LDL, las VLDL y otras lipoproteínas. Tiene una respuesta rápida y efectiva, pero es de alto costo y puede no ser suficiente para prevenir la ateromatosis prematura. Se recomienda considerarlo de manera temprana en pacientes con $\mathrm{HHF}^{8,10}$ y fue una alternativa en la paciente presentada, dada la refractariedad al manejo inicial.

Otra alternativa terapéutica es el trasplante de hígado, que al reemplazar el LDLR disfuncional, normaliza el colesterol. Aun no se sabe si esto es mejor que la combinación de fármacos con aféresis de lipoproteínas. ${ }^{10}$

Existen tres nuevos medicamentos que han demostrado ser eficaces y han sido aprobados por la FDA para la HHF en población adulta. El lomitapide es un inhibidor oral de la proteína de transferencia de triglicéridos liposomales. En niños no se ha establecido la seguridad y eficacia, pero una serie de casos mostró buenos resultados desde los cuatro años. ${ }^{11}$ El mipomersen inhibe la transcripción del gen que codifica la apolipoproteína $b$, y tampoco se ha establecido seguridad y eficacia en niños. Sin embargo, un ensayo clínico mostró su eficacia en la población pediátrica, con una tolerancia relativamente mala, al igual que en los adultos. ${ }^{12}$ Tanto el lopitamide como el mipomersen tienen como efecto adverso el aumento de las transaminasas y la esteatosis hepática. ${ }^{10,13} \mathrm{El}$ tercer fármaco es el evolocumab, un anticuerpo monoclonal que inhibe la PCSK9, una proteasa que degrada el LDLR. Hay datos que avalarían su seguridad y eficacia en niños desde el año de vida. ${ }^{14}$

Por último, es importante el consejo genético para diagnosticar la variante heterocigota en otros familiares y considerar el riesgo en la descendencia. $^{8}$

En el caso presentado se inició tratamiento con el esquema clásico recomendado; sin embargo, a los cuatro meses no se observó mejoría en el perfil

TABLA 2. Criterios diagnósticos de hipercolesterolemia homocigota familiar ${ }^{8}$

1. Confirmación genética de dos alelos mutantes en el locus genético LDLR, APOB, PCSK9 o LDLRAP1

2. Colesterol LDL $>500 \mathrm{mg} / \mathrm{dl}$ (sin tratamiento) o colesterol LDL $>300 \mathrm{mg} / \mathrm{dl}^{*}$ (con tratamiento), junto con alguno de los siguientes:

- Xantomas tendinosos o cutáneos antes de los 10 años.

- Niveles elevados de colesterol LDL (sin tratamiento), consistentes con hipercolesterolemia familiar heterocigota, en ambos padres.

Nota: para el diagnóstico deben cumplirse algunos de los dos criterios.

* Los niveles son de referencia, pero pueden ser más bajos, especialmente en niños o en pacientes recibiendo tratamiento, y no excluyen la patología. 
lipídico. Esto plantea la necesidad de considerar como opciones la aféresis, los fármacos nuevos y el trasplante hepático, dadas las implicancias en morbilidad y mortalidad que tiene en la paciente.

Este caso resalta la importancia del conocimiento y la pesquisa oportuna de los xantomas tuberosos como primera manifestación de la HHF. Los primeros en detectar sus manifestaciones clínicas serán los médicos de atención primaria, los pediatras y los dermatólogos, por lo que resulta esencial el reconocimiento, la derivación oportuna y el manejo multidisciplinario para prevenir la ateroesclerosis acelerada y la muerte precoz en el paciente y en sus familiares.

\section{REFERENCIAS}

1. Cruz PD, East C, Bergstresser PR. Dermal, subcutaneous, and tendon xanthomas: diagnostic markers for specific lipoprotein disorders. J Am Acad Dermatol. 1988; 19(1 Pt 1):95-111.

2. Raal FJ, Santos RD. Homozygous familial hypercholesterolemia: current perspectives on diagnosis and treatment. Atherosclerosis. 2012; 223(2):262-8.

3. Sacchi A, Olivares L, Leiro V. Xantomatosis y dislipoproteinemias. Dermatol Argent. 2013; 19(3):174-82.

4. Trent W. Xantomas. In: Bolognia J, Schaffer J, Cerroni L (eds). Dermatology. $4^{\text {th }}$ ed. China: Elsevier; 2018.Pág.1643.

5. Zak A, Zeman M, Slaby A, Vecka M. Xanthomas: clinical and pathophysiological relations. Biomed Pap Med Fac Univ Palacky Olomouc Czech Repub. 2014; 158(2):181-8.
6. Bansal M, Manchanda K, Pandey SS. Familial type IIa hyperlipoproteinemia presenting with different types of xanthomas. Indian J Paediatr Dermatol. 2014; 15(1):39-41.

7. Nordestgaard BG, Chapman MJ, Humphries SE, Ginsberg $\mathrm{HN}$, etal.Familialhypercholesterolaemiaisunderdiagnosed and undertreated in the general population: guidance for clinicians to prevent coronary heart disease: consensus statement of the European Atherosclerosis Society. Eur Heart J. 2013; 34(45):3478-90a.

8. Cuchel M, Bruckert E, Ginsberg HN, Raal FJ, et al. Homozygous familial hypercholesterolaemia: new insights and guidance for clinicians to improve detection and clinical management. A position paper from the Consensus Panel on Familial Hypercholesterolaemia of the European Atherosclerosis Society. Eur Heart J. 2014; 35(32):2146-57.

9. Sethuraman G, Thappa DM, Karthikeyan K. Intertriginous xanthomas-a marker of homozygous familial hypercholesterolemia. Indian Pediatr. 2000; 37(3):338.

10. France M. Homozygous familial hypercholesterolaemia: update on management. Paediatr Int Child Health. 2016; 36(4):243-7.

11. Ben-Omran T, Masana L, Kolovou G, Ariceta G, et al. RealWorld Outcomes with LomitapideUse in Paediatric Patients with Homozygous Familial Hypercholesterolaemia. Adv Ther. 2019; 36(7):1786-811.

12. Raal FJ, Braamskamp M, Selvey S, Sensinger C, Kastelein J. Pediatric experience with mipomersen as adjunctive therapy for homozygous familial hypercholesterolemia. J Clin Lipidol. 2016; 10(4):860-9.

13. Reiner $Z$. Treatment of children with homozygous familial hypercholesterolaemia. Eur J Prev Cardiol.2018;25(10):10957.

14. Campo R. Estudios clínicos con inhibidores de la PCSK9. Rev Colomb Cardiol. 2017; 24(2):13-8. 